\title{
Roughness and hydrophobicity studies of nanofiltration membranes using different modes of AFM
}

\author{
K. Boussu ${ }^{\mathrm{a}, *}$, B. Van der Bruggen ${ }^{\mathrm{a}}$, A. Volodin ${ }^{\mathrm{b}}$, J. Snauwaert $^{\mathrm{b}}$, C. Van Haesendonck ${ }^{\mathrm{b}}$, \\ C. Vandecasteele ${ }^{a}$ \\ ${ }^{a}$ Laboratory for Applied Physical Chemistry and Environmental Technology, Department of Chemical Engineering, K.U. Leuven, W. de Croylaan 46, \\ B-3001 Leuven, Belgium \\ ${ }^{\mathrm{b}}$ Laboratory of Solid-State Physics and Magnetism, Department of Physics and Astronomy, K.U. Leuven, Celestijnenlaan 200D, B-3001 Leuven, Belgium
} Received 19 October 2004; accepted 22 January 2005

Available online 24 March 2005

\begin{abstract}
Determination of the surface roughness by AFM is crucial to the study of particle fouling in nanofiltration. It is, however, very difficult to compare the different roughness values reported in the literature because of a lack in uniformity in the methods applied to determine surface roughness. AFM is used in both noncontact mode and tapping mode; moreover, the size of the scan area is highly variable. This study compares, for six different nanofiltration membranes (UTC-20, N30F, Desal 51HL, Desal 5DL, NTR7450, NF-PES-10), noncontact mode AFM with tapping mode AFM for several sizes of the scan area. Although the absolute roughness values are different for noncontact AFM and tapping mode AFM, no difference is found between the two modes of AFM in ranking the nanofiltration membranes with respect to their surface roughness. NTR 7450 and NF-PES-10 are the smoothest membranes, while the roughest surface can be found with Desal $51 \mathrm{HL}$ and Desal 5DL. UTC-20 and N30F are characterized by an intermediate roughness value. An increase in roughness with increasing scan area is observed for both AFM modes. Larger differences between the roughnesses of the membranes are obtained with tapping mode AFM because of the tapping of the tip on the surface. Phase imaging is an extension of tapping mode AFM, measuring the phase shift between the cantilever oscillation and the oscillation of the piezo driver. This phase shift reflects the interaction between the cantilever and the membrane surface. A comparison with contact angle measurements proves that a small phase shift corresponds to a large contact angle, representing a hydrophobic membrane surface.
\end{abstract}

(c) 2005 Elsevier Inc. All rights reserved.

Keywords: Nanofiltration; Tapping mode AFM; Noncontact AFM; Phase imaging; Contact angle; Surface roughness

\section{Introduction}

Nanofiltration is a promising technique for drinkingwater production from surface water and ground water. The main problem in using membrane technology is fouling, which results in an undesirable flux decline [1-3]. Fouling is partly due to suspended particulate matter. This type of fouling is very complex and is influenced by different parameters related to the solution itself (e.g., ionic strength and colloid

\footnotetext{
* Corresponding author. Fax: +32-16-32-29-91.

E-mail address: katleen.boussu@cit.kuleuven.ac.be (K. Boussu).
}

concentration), but the surface morphology of the membrane also plays a crucial role.

According to Elimelech and co-workers [4-7], colloidal fouling can be correlated with the surface roughness of nanofiltration and reverse osmosis membranes. Their experiments showed that the permeate flux of the rougher composite polyamide membranes was substantially lower than the permeate flux of the smoother cellulose acetate membranes. This was explained by atomic force microscopy (AFM) images showing that in the initial stages of fouling, the colloidal particles preferentially accumulated in the "valleys" of rough membranes, resulting in "valley clogging" and hence in a more severe flux decline. 
Also, in the concentration of apple and pear juices by nanofiltration, Warczok et al. [8] concluded that smoother membranes showed a lower yield of irreversible fouling of yeast particles. However, no correlation between particle fouling and membrane roughness was found by Van der Bruggen et al. [9]: experiments seemed to support the assumption that particle fouling is mainly determined by the hydrophobicity of the membranes.

In the case of filtrating a dilute $\mathrm{NaCl}$ solution, Hirose et al. [10] found that an approximately linear relationship existed between the surface roughness of reverse osmosis membranes and the membrane flux. In this case the skin layer unevenness is regarded as an enlargement of the effective membrane surface area.

Research in the past years has demonstrated the power of AFM for studying colloidal fouling. AFM probes the surface of a sample with a sharp tip, located at the free end of a cantilever $[11,12]$. Forces between the tip and the sample surface cause the cantilever to bend. This cantilever deflection is detected optically by focusing a laser beam onto the back of a reflective cantilever. As the tip scans the surface of the sample, moving up and down with the contour of the surface, the laser beam is deflected off the attached cantilever into a dual element photodiode (the beam-bounce method). This photodetector measures the difference in light intensities between the upper and lower photodiodes and then converts this signal into a voltage. This method enables a computer to generate a three-dimensional map of the surface topography.

There are three commonly used AFM techniques: contact mode, noncontact mode, and tapping mode. In contact mode, the tip scans the sample in close contact with the surface. This means that the interatomic force between the sample and the tip is repulsive, with a typical value of $10^{-7} \mathrm{~N}$. Problems with contact mode are caused by excessive tracking forces applied to the sample by the probe. Therefore, contact mode AFM cannot be applied to soft surfaces, such as polymeric membranes.

In situations where tip contact might alter the sample, noncontact mode is used. In this mode the tip moves about 50-150 ̊ above the sample surface. Attractive interatomic forces between the tip and the sample are detected, and topographic images are constructed by scanning the tip above the surface. The attractive forces from the sample are, however, substantially weaker $\left(10^{-13} \mathrm{~N}\right)$ than the forces used in contact mode. Therefore the cantilever is driven to vibrate near its resonant frequency by means of a piezoelectric element and changes in the resonant frequency as a result of tip-surface force interaction are measured (dynamic detection method). Problems with noncontact mode can be caused by a contaminant layer (present on the sample), which interferes with the cantilever oscillation and results in low resolution.

Tapping mode AFM allows high-resolution topographic imaging of sample surfaces by alternately bringing the tip into contact with the surface to provide high resolution and then lifting it off the surface to avoid dragging the tip across the surface. Tapping mode imaging is again implemented by oscillating the cantilever assembly at or near the cantilevers resonant frequency using a piezoelectric crystal. The piezo motion causes the cantilever to oscillate with a large amplitude (typically greater than $20 \mathrm{~nm}$ ) when the tip is not in contact with the surface. The oscillating tip is then moved toward the surface until it begins to gently touch or tap the surface. During scanning, the vertically oscillating tip alternately contacts the surface and lifts off, generally at a frequency of 50,000-500,000 cycles per second. As the oscillating cantilever begins to intermittently contact the surface, the cantilever oscillation is reduced due to energy loss caused by the tip contacting the surface. The reduction in oscillation amplitude is used to identify and measure surface features.

A powerful extension of tapping mode AFM is phase imaging. As mentioned above, in tapping mode AFM, the cantilever is excited into resonance oscillation with a piezoelectric driver and the oscillation amplitude is used as a feedback signal to measure topographic variations of the sample surface. In phase imaging, the phase shift of the cantilever oscillation, relative to the signal sent to the cantilever's piezo driver, is simultaneously monitored and recorded. This phase shift is very sensitive to local variations in the material properties.

One of the advantages of AFM is that it allows imaging of insulators and semiconductors as well as electrical conductors. That is why AFM has a lot of applications [13], such as analyzing ionic crystals, DNA and RNA [14,15], and red blood cells [16]. AFM has also been used to study the roughness of polymeric [17-31] and ceramic membranes [32,33]. For ultrafiltration and microfiltration membranes AFM can be used not only to measure the roughness, but also to study the size and the shape of pores on the surface [18-20,23-25, $27,29,30]$. For the smaller pores of nanofiltration and reverse osmosis, however, great care is needed in the interpretation of pore diameters (obtained by AFM) because of convolution effects between the tip and the pore [21,22]. Another application of AFM in the membrane field is the direct measurement of the force of adhesion between a particle (such as silica or polystyrene) and the membrane surface by immobilizing the particle at the end of the cantilever. This can be a great benefit to membrane technologists, as it makes it possible to predict fouling without process measurements [22, 24,34].

Although AFM is widely used to characterize membranes, it is very difficult to compare different measurements. In the literature one finds roughness values obtained by several modes of AFM (mentioned or not), determined for different scan areas (mentioned or not). A detailed and systematic comparison between different AFM modes and between different scan areas has not been presented before. Phase imaging has never been studied. 
The aim of this paper is to study the influence of the AFM mode and the influence of the size of the scan area on the roughness values obtained for six different nanofiltration membranes. AFM phase imaging is presented as a reliable alternative for contact angle measurements. Concerning particle fouling, this study forms the basis to find a potential correlation between the membrane performance and the roughness in future experiments.

\section{Materials and methods}

Six nanofiltration membranes were studied: UTC-20 (Toray Ind), Desal 5DL (Osmonics), Desal 51HL (Osmonics), N30F (Nadir), NTR 7450 (Nitto-Denko), and NF-PES10 (Nadir). The characteristics of the membranes are summarized in Table 1. The MWCO (molecular weight cut-off) of the different membranes (given by the manufacturer) is checked by doing cross-flow filtration experiments with a mixture of polyethylene glycols. These experimentally obtained cut-offs, together with the MWCO of the manufacturers, are also mentioned in Table 1. It seems that the MWCO (measured with the polyethylene glycol) accords well with the data of the manufacturer, except for N30F, NF-PES-10, and NTR 7450. N30F and NF-PES-10, seem to have larger pores (respectively $580 \mathrm{Da}$ instead of $400 \mathrm{Da}$ and $1200 \mathrm{Da}$ instead of $1000 \mathrm{Da}$ ). On the other hand, the pores of NTR 7450 are much smaller than stated by the manufacturer (310 Da instead of 600-800 Da).

Noncontact AFM was performed with a M5 AFM system (Park Scientific Instruments, currently VEECO). The cantilever was made out of $\mathrm{Si}$ with a spring constant of $3.2 \mathrm{~N} / \mathrm{m}$ and a nominal tip apex radius of $10 \mathrm{~nm}$. After scanning, the images were flattened with order 2 . This implies that a least-squares fit is made to the data in the scanned region using a second-order polynomial; the resulting best fit is then subtracted from the image. Flattening was done to remove curvature and slope from an image. After flattening, the RMS roughness (root-mean-squared roughness) was calculated. The RMS roughness $R_{\mathrm{rms}}$ is given by the standard deviation of the data,

$R_{\mathrm{rms}}=\sqrt{\frac{\sum_{n=1}^{N}\left(z_{n}-\bar{z}\right)^{2}}{N-1}}$,

where $\bar{z}$ is the average of the $z$ values within the given area, $z_{n}$ is the current $z$ value, and $N$ is the number of data points within the given area. Other definitions can also be used to characterize the roughness, such as the mean roughness (the mean value of the surface relative to the center plane) or the peak-to-valley distance (the distance between the highest data point and the lowest data point of the surface). However, these definitions are less accurate than the RMS roughness. In the peak-to-valley distance only two points of the scanned surface (namely the highest and the lowest) are taken into account, which is not very representative of the whole surface. A comparison between the RMS and the mean roughness shows that the latter takes less account of the variations of the low frequencies. Hence the RMS roughness is the most accurate and will be used in the rest of this paper.

Tapping mode AFM images, as well as AFM phase images, were obtained with a Nanoscope III scanning probe microscope (Digital Instruments, Santa Barbara, California). The SPM probe used was a $\mathrm{SiO}_{2}$ noncontact SPM probe (Nanosensors) with a spring constant of $40 \mathrm{~N} / \mathrm{m}$, a resonant frequency of $190 \mathrm{kHz}$, and a typical radius of $10 \mathrm{~nm}$. After scanning, the images were flattened with order 2 and the RMS value of the roughness was calculated by Eq. (1). The RMS value was also calculated for the phase shift $\Theta_{\mathrm{rms}}$ by the equation

$\Theta_{\mathrm{rms}}=\sqrt{\frac{\sum_{n=1}^{N}\left(\theta_{n}-\bar{\theta}\right)^{2}}{N-1}}$,

where $\bar{\theta}$ is the average of the $\theta$ values within the given area, $\theta_{n}$ is the current $\theta$ value, and $N$ is the number of data points within the given area.

For noncontact AFM, as well as for tapping mode AFM, the analyses were executed on a dry sample in an atmosphere with relative humidity $32 \%$. Measuring a dry sample has two advantages compared to measuring a wet surface. First of

Table 1

Characteristics of the nanofiltration membranes

\begin{tabular}{|c|c|c|c|c|c|c|}
\hline & \multicolumn{6}{|l|}{ Membrane } \\
\hline & Desal 5DL & Desal 51HL & UTC-20 & $\mathrm{N} 30 \mathrm{~F}$ & NTR 7450 & NF-PES-010 \\
\hline $\mathrm{MWCO}^{\mathrm{a}}(\mathrm{Da})$ & $150-300$ & $150-300$ & 180 & 400 & $600-800$ & 1000 \\
\hline Max temp. $\left({ }^{\circ} \mathrm{C}\right)$ & 90 & 50 & 35 & 95 & 40 & 95 \\
\hline Max pressure (bar) & 40 & 40 & 15 & - & 30 & - \\
\hline $\mathrm{pH}$ range & $1-11$ & $3-9$ & $3-10$ & $0-14$ & $2-14$ & $0-14$ \\
\hline
\end{tabular}

a MWCO: MWCO of the membranes as given by the manufacturer.

b MWCO: MWCO of the membranes as experimental obtained by using a mixture of polyethylene glycols. 
all, it is easier to make a distinction between a hydrophobic and a hydrophilic surface by measuring a dry surface (with a hydrophilic tip). On a hydrophilic dry surface, the relative humidity will deliver an additional capillary force, which will not be present on a wet surface. Because of the absence of the capillary force in measuring a wet surface, it is not possible to distinguish between a hydrophobic and a hydrophilic surface. The second reason for choosing a dry surface is the measurement time measuring a wet surface takes 5-10 times longer than measuring a dry surface.

In all cases, the roughness and the phase shift were measured for five different scan areas: $0.5 \times 0.5 \mu \mathrm{m}^{2}, 1 \times 1 \mu \mathrm{m}^{2}$, $3 \times 3 \mu \mathrm{m}^{2}, 5 \times 5 \mu \mathrm{m}^{2}$, and $10 \times 10 \mu \mathrm{m}^{2}$. The first scan was always made for the largest area $\left(10 \times 10 \mu \mathrm{m}^{2}\right)$ and then this area was reduced till the smallest area of $0.5 \times 0.5 \mu \mathrm{m}^{2}$ was reached. In the case of noncontact AFM, each measurement was done three times to obtain a mean value of the RMS roughness. The standard deviation of the different measurements was small, ranging from $0.8 \AA$ on the smooth surfaces to $6.2 \AA$ on the rough surfaces. Hence, the distributions of the roughness values were small, indicating that the results are reproducible.

Contact angle measurements were performed with a DSA $10 \mathrm{Mk} 2$ drop shape analysis system (Krüss) in a three-phase system consisting of the membrane surface, air, and a drop of water. The sessile drop method was chosen and the contact angle was measured in an equilibrium mode. This means that a droplet of water is placed on the membrane surface, after which the contact angle between the droplet and the membrane surface is calculated by the computer program. In the case of a hydrophilic surface the droplet of water will spread out over the surface, resulting in a small contact angle; the opposite is true in the case of a hydrophobic surface. Each contact angle was measured 10-15 times and an average value was calculated. The contact angle of the wet, clean membrane was determined for all membranes.

\section{Results and discussion}

\subsection{Roughness measurements with noncontact AFM and tapping mode AFM}

The roughness measurements obtained with noncontact AFM and tapping mode AFM are given in Tables 2 and 3, respectively. From these results it appears that the scanned area plays a significant role: the larger the scanned area, the larger the roughness. Fig. 1 illustrates this effect for the noncontact AFM images of the Desal 5DL membrane for four different membrane areas. The phenomenon of increasing roughness with increasing scan area can be related to the dependency of the roughness on the spatial wavelength of the scanned area or the frequency. For a small surface area, only the roughness of the "higher" frequencies is measured. When a larger surface area is scanned the roughness caused by additional lower frequencies also has to be taken into account. This results in a larger roughness value when a larger surface area is scanned. Another explanation for the increasing roughness with increasing scan size may be the formation of a fractal structure on the membrane surface when polymers are assembled to nodules or aggregates of nodules. So, when the scan size is changed, it is possible to get a different surface topography, resulting in a different roughness. Therefore it is crucial that the same scan size range is used when comparing the surface roughness for different samples.

For noncontact AFM, as well as for tapping mode AFM, the arrangement of the six membranes according to their surface roughness is the same,

NTR $7450<$ NF-PES-10 $<$ N30F $<$ UTC-20 $<$ Desal $51 \mathrm{HL}<$ Desal 5DL, with NTR 7450 the smoothest membrane.

Figs. 2 and 3 show noncontact and tapping mode AFM images, respectively, of a very smooth membrane (NF-PES$10)$ and a very rough membrane (Desal 5DL) taken for an area of $3 \times 3 \mu \mathrm{m}^{2}$.

Table 2

Roughness measurements (in Ångström) with noncontact AFM for different areas $\left(0.5 \times 0.5 \mu \mathrm{m}^{2}, 1 \times 1 \mu \mathrm{m}^{2}, 3 \times 3 \mu \mathrm{m}^{2}, 5 \times 5 \mu \mathrm{m}^{2}\right.$, and $\left.10 \times 10 \mu \mathrm{m}^{2}\right)$

\begin{tabular}{lccllrr}
\hline Area & NTR 7450 & NF-PES-10 & N30F & UTC-20 & Desal 51HL \\
\hline $0.5 \times 0.5 \mu \mathrm{m}^{2}$ & 8 & 8 & 13 & 22 & 26 & Desal 5DL \\
$1 \times 1 \mu \mathrm{m}^{2}$ & 9 & 13 & 25 & 26 & 52 \\
$3 \times 3 \mu \mathrm{m}^{2}$ & 15 & 24 & 34 & 46 & 59 & 58 \\
$5 \times 5 \mu \mathrm{m}^{2}$ & 18 & 27 & 39 & 48 & 62 & 109 \\
$10 \times 10 \mu \mathrm{m}^{2}$ & 31 & 38 & 55 & 68 & 112 & 128 \\
\hline
\end{tabular}

Table 3

Roughness measurements (in Ångström) with tapping mode AFM for different areas $\left(0.5 \times 0.5 \mu \mathrm{m}^{2}, 1 \times 1 \mu \mathrm{m}^{2}, 3 \times 3 \mu \mathrm{m}^{2}, 5 \times 5 \mu \mathrm{m}^{2}\right.$, and $\left.10 \times 10 \mu \mathrm{m}^{2}\right)$

\begin{tabular}{lcccccc}
\hline Area & NTR 7450 & NF-PES-10 & N30F & UTC-20 & Desal 51HL \\
\hline $0.5 \times 0.5 \mu \mathrm{m}^{2}$ & 5 & 8 & 9 & 20 & 37 & Desal 5DL \\
$1 \times 1 \mu \mathrm{m}^{2}$ & 7 & 11 & 16 & 28 & 59 & 108 \\
$3 \times 3 \mu \mathrm{m}^{2}$ & 21 & 22 & 39 & 56 & 64 & 132 \\
$5 \times 5 \mu \mathrm{m}^{2}$ & 23 & 25 & 41 & 48 & 93 & 139 \\
$10 \times 10 \mu \mathrm{m}^{2}$ & 32 & 35 & 49 & 88 & 118 & 147 \\
\hline
\end{tabular}


(a) $0.5 \mu \mathrm{m} \times 0.5 \mu \mathrm{m}$

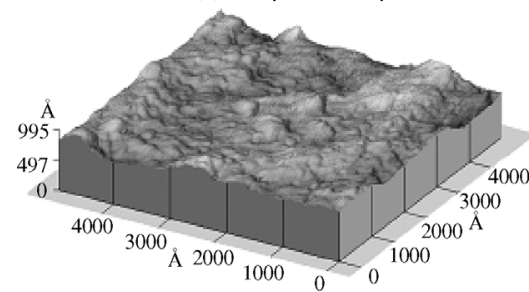

(c) $5 \mu \mathrm{m} \times 5 \mu \mathrm{m}$

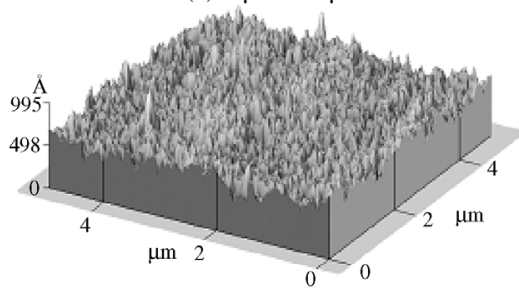

(b) $1 \mu \mathrm{m} \times 1 \mu \mathrm{m}$

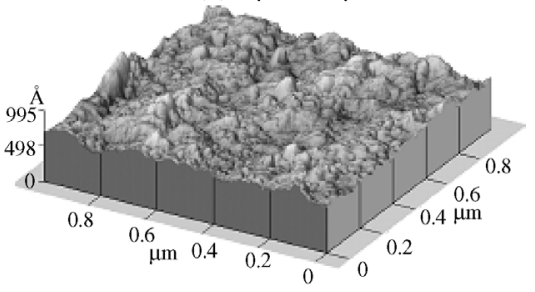

(d) $10 \mu \mathrm{m} \times 10 \mu \mathrm{m}$

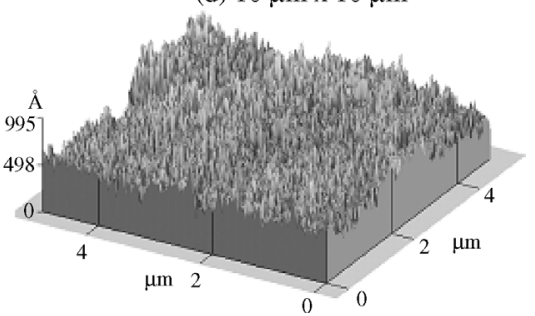

Fig. 1. Noncontact AFM images of Desal 5DL for four different scan areas: (a) $0.5 \times 0.5 \mu \mathrm{m}^{2}$, (b) $1 \times 1 \mu \mathrm{m}^{2}$, (c) $5 \times 5 \mu \mathrm{m}^{2}$, (d) $10 \times 10 \mu \mathrm{m}^{2}$. Note that the $Z$-scale is always $995 \AA$.

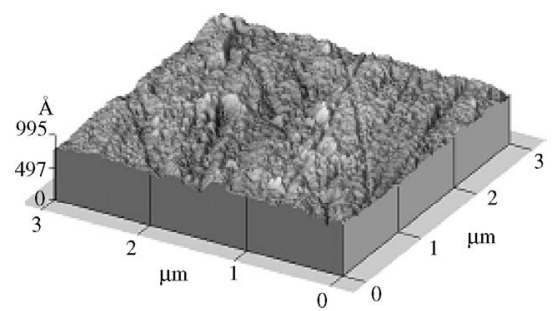

(a) NF-PES-10

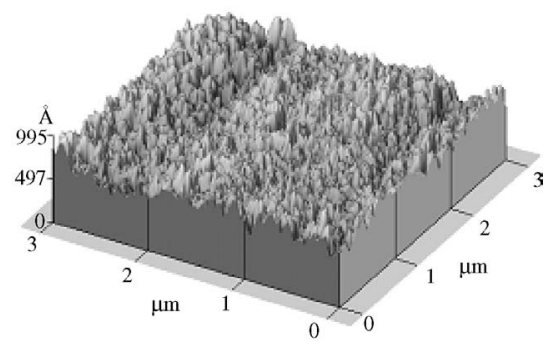

(b) Desal 5DL

Fig. 2. Noncontact AFM images of (a) NF-PES-10 and (b) Desal 5DL. Note that the $X$ and $Y$ dimensions are both $3 \mu \mathrm{m}$, while the $Z$-scale is $995 \AA$.

The roughness values are very different for the six nanofiltration membranes, varying from 8 to $52 \AA$ in noncontact mode and from 5 to $68 \AA$ in tapping mode for a scanned area of $0.5 \times 0.5 \mu \mathrm{m}^{2}$. This implies that the difference between the smoothest membrane and the roughest membrane is the largest in tapping mode AFM (63 A versus $44 \AA$ in non-contact mode).

Fig. 4 compares roughnesses obtained in noncontact AFM and in tapping mode AFM for a scanned area of $0.5 \times 0.5 \mu \mathrm{m}^{2}$ for the six nanofiltration membranes, together with the bisector of the graph. The data points for the smoother membranes are located under the bisector, meaning that the roughness measured in tapping mode is smaller than in noncontact mode. The opposite is observed for the

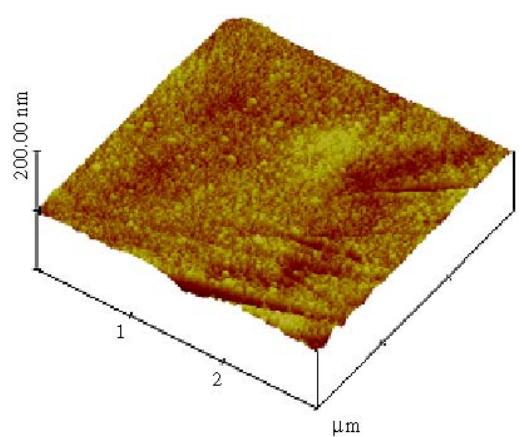

(a) NF-PES-10

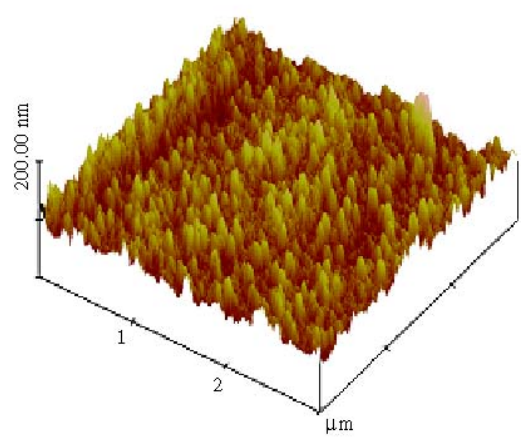

(b) Desal 5DL

Fig. 3. Tapping mode AFM images of (a) NF-PES-10 and (b) Desal 5DL. Note that the $X$ and $Y$ dimensions are both $3 \mu \mathrm{m}$, while the $Z$-scale is $200 \mathrm{~nm}$.

rougher membranes Desal 51HL and Desal 5DL. So tapping mode AFM results in a larger contrast between the roughnesses of the membranes. Similar conclusions can be drawn for the other scanned areas.

This phenomenon is intrinsic to the method of measuring roughness in tapping mode AFM. In tapping mode the tip (attached to the cantilever) taps the scanned surface in contrast to noncontact AFM, where the distance between the tip 
Table 4

RMS of the phase shift measurements (in ${ }^{\circ}$ ) with tapping mode AFM for different areas $\left(0.5 \times 0.5 \mu \mathrm{m}^{2}, 1 \times 1 \mu \mathrm{m}^{2}, 3 \times 3 \mu \mathrm{m}^{2}, 5 \times 5 \mu \mathrm{m}^{2}\right.$, and $\left.^{2} 10 \times 10 \mu \mathrm{m}^{2}\right)$

\begin{tabular}{|c|c|c|c|c|c|c|}
\hline Area & $\mathrm{N} 30 \mathrm{~F}$ & NTR 7450 & NF-PES-10 & Desal 5DL & UTC-20 & Desal 51HL \\
\hline $0.5 \times 0.5 \mu \mathrm{m}^{2}$ & 0.91 & 1.32 & 1.65 & 1.67 & - & 4.57 \\
\hline $1 \times 1 \mu \mathrm{m}^{2}$ & 0.93 & 1.35 & 2.87 & 2.48 & 2.16 & 4.44 \\
\hline $3 \times 3 \mu \mathrm{m}^{2}$ & 1.83 & 2.27 & 3.02 & 3.57 & 4.10 & 6.63 \\
\hline $10 \times 10 \mu \mathrm{m}^{2}$ & 3.55 & 5.22 & 6.78 & 6.49 & 6.64 & 9.50 \\
\hline
\end{tabular}

Note. - : no data available.

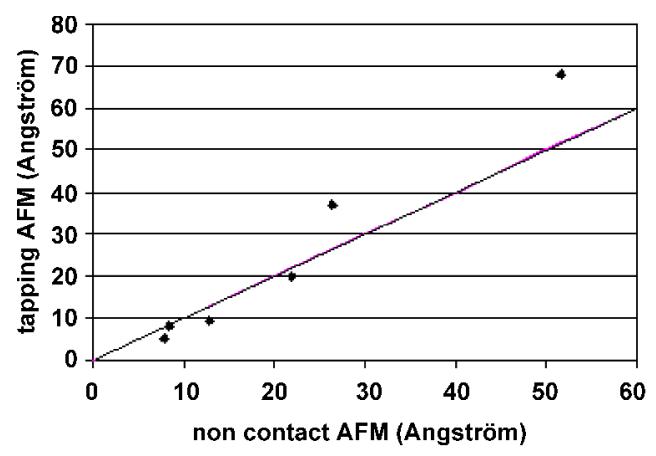

Fig. 4. Comparison between the roughness measured in noncontact AFM and the roughness measured in tapping mode AFM for an area of $0.5 \times 0.5 \mu \mathrm{m}^{2}$.

and the surface is approximately $100 \AA$. When a contaminant layer (e.g., a few monolayers of condensed water) covers the surface, an AFM operating in tapping mode penetrates the layer to image the underlying surface. In noncontact mode, however, the AFM tip cannot penetrate the water layer and hence it will image the surface of the water layer. This results in a smaller difference between the roughnesses of the different nanofiltration membranes in noncontact AFM.

\subsection{Phase shift measurements with tapping mode AFM}

Using tapping mode AFM, the phase shift can be measured simultaneously. The phase shift is derived from the difference in phase angle between the freely oscillating cantilever in air and the cantilever oscillation during scanning. The phase scaling convention is based on the freely resonating cantilever in air. The phase shift is zero when there is no interaction between the tip or the cantilever and the sample surface. However, in the case of a tip-sample interaction, a phase lag is induced if the interaction is attractive and a phase advanced appears if the interaction is repulsive. Areas in a phase image exhibiting a phase lag with respect to the free oscillation are shown darkened; regions of advanced phase are lightened. Fig. 5 represents a twodimensional phase image of the NF-PES-10 membrane and the Desal 51HL membrane taken for an area of $3 \times 3 \mu \mathrm{m}^{2}$.

The results of the measured RMS phase shifts for the different membranes and for the different scanned areas are represented in Table 4. From this table it becomes clear that the six membranes can be divided into two groups. The first group consists of the membranes made of polyethersulfone

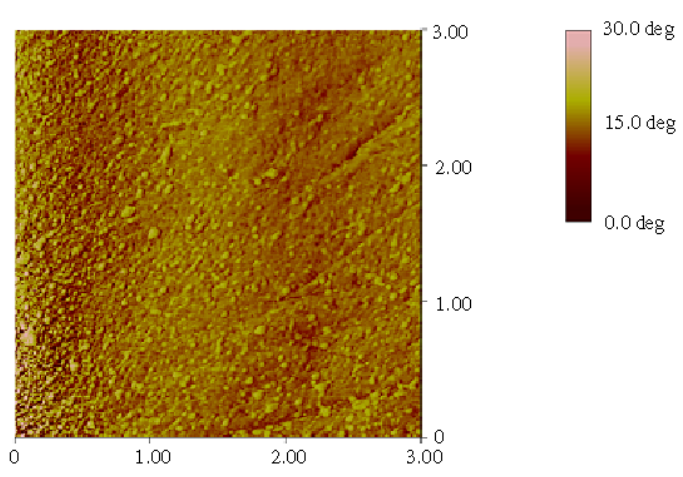

(a) NF-PES-10

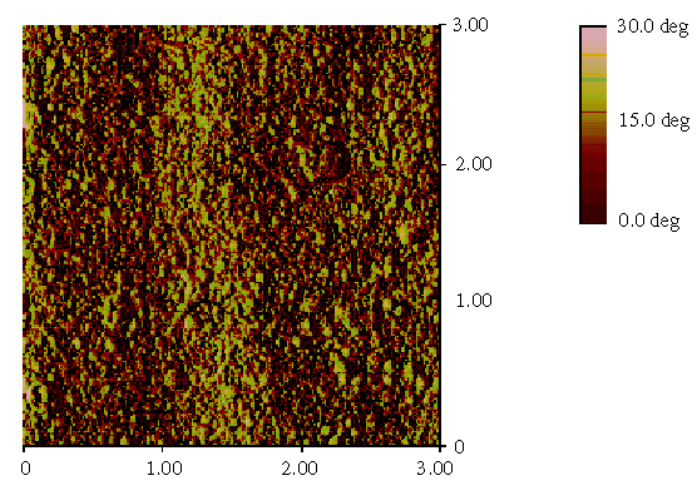

(b) Desal 51HL

Fig. 5. Phase images of (a) NF-PES-10 and (b) Desal 51HL for an area of $3 \times 3 \mu \mathrm{m}^{2}$.

(N30F, NTR 7450, and NF-PES-10), which have a smaller interaction with the tip, resulting in a small phase shift. More interaction (and hence larger phase shifts) occurs between the tip and the membranes made of polyamide (Desal 5DL, Desal 51HL, and UTC-20), which form the second group.

Because the experiments were carried out with a hydrophilic silicon tip, one can observe whether the interaction between the tip and the sample is hydrophilic or hydrophobic. A hydrophilic surface interacts strongly with a hydrophilic tip, resulting in a large phase shift. A hydrophobic surface does not interact very strongly with a hydrophilic tip, resulting in a small phase shift. Consequently one can divide the six membranes in two groups with respect to their surface hydrophobicity. The more hydrophobic group consists of the membranes with the smaller phase shifts (N30F, NTR 7450, and NF-PES-10), while the hydrophilic 
Table 5

Contact angles $\left(\right.$ in $^{\circ}$ ) measured for the six nanofiltration membranes

\begin{tabular}{ll}
\hline Membrane & Contact angle $\left({ }^{\circ}\right)$ \\
\hline UTC-20 & $36.4 \pm 7.7$ \\
Desal 5DL & $43.8 \pm 2.5$ \\
Desal 51HL & $47.1 \pm 8.3$ \\
NTR 7450 & $69.6 \pm 5.9$ \\
NF-PES-10 & $72.1 \pm 3.7$ \\
N30F & $88.4 \pm 1.9$ \\
\hline
\end{tabular}

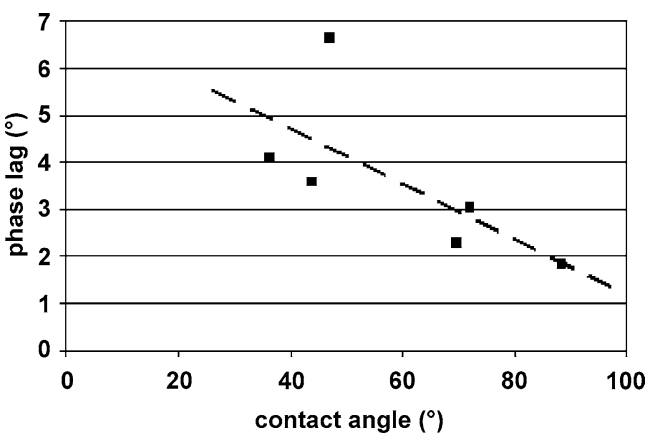

Fig. 6. Comparison between the phase shift (measured in tapping mode AFM) and the contact angle for an area of $3 \times 3 \mu \mathrm{m}^{2}$.

group contains the other three membranes (Desal 5DL, Desal 51HL, and UTC-20), with the larger phase shifts.

These phase shifts can be compared with the measured contact angles in Table 5. Contact angles are an accepted method for determining the hydrophobicity of a surface, in the sense that a larger contact angle corresponds to a more hydrophobic material.

The contact angles are compared with the phase shifts for the different nanofiltration membranes in Fig. 6. These results indicate that a large contact angle corresponds to a small phase shift. The only exception to this is the Desal 51HL membrane, which shows a very large phase shift (for all the scanned areas) compared to the contact angle. This indicates that a very strong specific interaction occurs between the hydrophilic silicon tip and the Desal 51HL membrane, resulting in a very large phase shift.

\section{Conclusions}

Noncontact AFM and tapping mode AFM both predict the same order of surface roughness. However, a comparison between different membranes and different AFM modes is only valid when considering the same surface area.

Although tapping mode AFM is more time-consuming, it gives more realistic results than noncontact AFM. An additional advantage of tapping mode AFM is the simultaneous measurement of the phase shift, a valuable alternative for the contact angle to study the hydrophobicity of the surface.

\section{Acknowledgment}

This research was financed with a fellowship from IWT (Institute for the Promotion of Innovation by Science and Technology in Flanders).

\section{References}

[1] L.D. Nghiem, A.I. Schäfer, T.D. Waite, Desalination 147 (2002) 269.

[2] C. Jarusutthirak, G. Amy, J.P. Croué, Desalination 145 (2002) 247.

[3] M. Manttari, L. Puro, J. Nuortila-Jokinen, M. Nyström, J. Membr. Sci. 165 (2000) 1.

[4] E.M. Vrijenhoek, S. Hong, M. Elimelech, J. Membr. Sci. 188 (2001) 115.

[5] X. Zhu, M. Elimelech, Environ. Sci. Technol. 31 (1997) 3654.

[6] X. Zhu, M. Elimelech, J. Environ. Eng. 121 (1995) 884.

[7] M. Elimelech, X. Zhu, A.E. Childress, S. Hong, J. Membr. Sci. 127 (1997) 101.

[8] J. Warczok, M. Ferrando, F. Lopez, C. Güell, J. Food Eng. 63 (2004) 63.

[9] B. Van der Bruggen, D. Segers, C. Vandecasteele, L. Braeken, A. Volodin, C. Van Haesendonck, Sep. Purif. Technol. 36 (2004) 203.

[10] M. Hirose, H. Ito, Y. Kamiyama, J. Membr. Sci. 121 (1996) 20.

[11] H.K. Wickramasinghe, AIP Conference Proceedings 241, American Institute of Physics, Santa Barbara, 1992.

[12] R. Wiesendanger, Scanning Probe Microscopy and Spectroscopy: Methods and Applications, Cambridge Univ. Press, Cambridge, UK, 1994.

[13] N.C. Santos, M. Castanho, Biophys. Chem. 107 (2004) 133.

[14] A.N. Round, M.J. Miles, Nanotechnology 15 (2004) S176.

[15] A.G. Wu, L.H. Yu, Z.A. Li, H.M. Yang, E.K. Wang, Anal. Biochem. 325 (2004) 293.

[16] P. Zachee, J. Snauwaert, P. Vandenberghe, L. Hellemans, M. Boogaerts, Br. J. Haematol. 95 (1996) 472.

[17] K.C. Khulbe, T. Matsuura, Polymer 41 (2000) 1917.

[18] A. Bessières, M. Meireles, R. Coratger, J. Beauvillain, V. Sanchez, J. Membr. Sci. 109 (1996) 271.

[19] J.Y. Kim, H.K. Lee, S.C. Kim, J. Membr. Sci. 163 (1999) 159.

[20] W.R. Bowen, N. Hilal, R.W. Lovitt, P.M. Williams, J. Membr. Sci. 110 (1996) 233

[21] S. Kwak, M. Yeom, I.J. Roh, D.Y. Kim, J. Kim, J. Membr. Sci. 132 (1997) 183.

[22] W.R. Bowen, T.A. Doneva, Desalination 129 (2000) 163.

[23] W.R. Bowen, T.A. Doneva, Surf. Interface Anal. 29 (2000) 544.

[24] W.R. Bowen, T.A. Doneva, J. Austin, G. Stoton, Colloids Surf. B Biointerfaces 27 (2002) 103.

[25] I.H. Huisman, P. Pradanos, A. Hernandez, J. Membr. Sci. 179 (2000) 79.

[26] V. Freger, J. Gilron, S. Belfer, J. Membr. Sci. 209 (2002) 283.

[27] T. Gumi, M. Valiente, K.C. Khulbe, C. Palet, T. Matsuura, J. Membr. Sci. 212 (2003) 123

[28] M. Davey, K. Landman, J.M. Perera, G.W. Stevens, N.D. Lawrence, M. Iyer, AIChE J. 50 (2004) 1431.

[29] A.K. Ho, J.M. Perera, D.E. Dunstan, G.W. Stevens, M. Nystrom, AIChE J. 45 (1999) 1434.

[30] P. Vaisanen, M.R. Bird, M. Nyström, Food Bioproducts Process. 80 (2002) 98.

[31] S. Kwak, D.W. Ihm, J. Membr. Sci. 158 (1999) 143.

[32] M. Vilaseca, E. Mateo, L. Palacio, P. Pradanos, A. Hernandez, A. Paniagua, J. Coronas, J. Santamaria, Micropor. Mesopor. Mater. 71 (2004) 33.

[33] J. Marchese, C. Almandoz, M. Amaral, L. Palacio, J.I. Calvo, P. Pradanos, A. Hernandez, Bol. Soc. Española Ceram. Vidr. 39 (2000) 215.

[34] N. Hilal, W.R. Bowen, Desalination 150 (2002) 289. 